\title{
Neurological Sequelae of Sepsis: I) Septic Encephalopathy
}

\author{
Thomas M. Ringer ${ }^{*}, 1$, Hubertus Axer ${ }^{1,2}$, Bernd F.M. Romeike ${ }^{3}$, Jan Zinke ${ }^{1,2}$, Frank Brunkhorst ${ }^{2,4}$, \\ Otto W. Witte ${ }^{1,2}$ and Albrecht Günther ${ }^{1,2}$
}

\author{
${ }^{I}$ Hans Berger Clinic for Neurology, Jena University Hospital, Friedrich-Schiller-University, Erlanger Allee 101, D- \\ 07747 Jena, Germany \\ ${ }^{2}$ CSCC - Center for Sepsis Control and Care, Jena University Hospital, Friedrich-Schiller-University, Erlanger Allee \\ 101, D-07747 Jena, Germany \\ ${ }^{3}$ Department of Neuropathology, Friedrich-Schiller-University Jena, Erlanger Allee 101, D-07747 Jena, Germany \\ ${ }^{4}$ Paul-Martini-Clinical Sepsis Research Unit, Jena University Hospital, Friedrich-Schiller-University, Erlanger Allee \\ 101, D-07747 Jena, Germany
}

\begin{abstract}
Septic encephalopathy (SE) or sepsis-associated delirium is the most common encephalopathy in ICU patients. It is defined by brain dysfunction due to systemic inflammatory response syndrome and extracranial infection. Clinically, acute impairment in level of consciousness and confusion are primarily defining symptoms. Precise clinical evaluation of brain function is crucial, although the necessary diagnostic tools are limited and require further verification in clinical studies. Therefore, SE is often underestimated and not frequently diagnosed. This review gives an overview of clinical features, epidemiological data, pathophysiological processes, imaging and neuropathological findings as well as diagnostic and therapeutic approaches in SE patients to characterize this severe neurological complication of sepsis.
\end{abstract}

Keywords: Encephalopathy, delirium, sepsis, SIRS.

\section{INTRODUCTION}

With the rapid advancement of critical care medicine in the last decades a variety of additional complications arose by evolution of controlling organ failures that formerly lead to death. Consensus criteria have been established by the American College of Chest Physicians and Society of Critical Care Medicine in 1992 defining systemic inflammatory response syndrome (SIRS), sepsis, severe sepsis, septic shock, bacteremia, and multiple organ dysfunction syndrome (MODS) as specified entities of the disease (Table 1). SIRS is defined as "the systemic inflammatory response to a variety of severe clinical insults" manifested by at least two of the following: (a) hyperthermia or hypothermia, (b) tachycardia, (c) tachypnea or hyperventilation, or (d) leukocytosis or leukopenia [1]. Later on several international organizations declared "SIRS can be triggered by a variety of infectious and noninfectious conditions" [2]. However, it can be simplified by differentiation of two main categories of SIRS: sepsis (defined as SIRS with infection) and noninfectious inflammation (e.g. due to burns, pancreatitis, acute respiratory distress syndrome, surgery, and trauma). Interestingly, SIRS seems to develop in two phases with complex pathophysiology: an initial pro-inflammatory state and a following anti-inflammatory state [3]. Severe sepsis is characterized by at least one organ dysfunction or

*Address correspondence to this author at the Hans Berger Clinic for Neurology, Jena University Hospital, Friedrich-Schiller-University, Erlanger Allee 101, D-07747 Jena, Germany; Tel: ++49 +3641 9323401; Fax: ++49+3641 9323402; E-mail: thomas.ringer@med.uni-jena.de hypoperfusion and septic shock describes severe sepsis associated with hypotension that is resistant to adequate fluid resuscitation. All these entities can involve different organs leading to MODS, if there are two or more organs impaired in an acutely ill patient with need for therapeutic intervention to maintain homeostasis.

Table 1. Consensus Definitions of a Spectrum of Clinical Entities that Result in Organ Failure

\begin{tabular}{l} 
Systemic Inflammatory Response Syndrome (SIRS): \\
An inflammatory response to a wide variety of clinical insults manifested \\
by two or more of the following symptoms: \\
- temperature $>38^{\circ} \mathrm{C}$ or $<36^{\circ} \mathrm{C}$ \\
- heart rate $>90 / \mathrm{min}$ \\
- respiratory rate $>20 / \mathrm{min}$ or $\mathrm{PaCO}_{2}<32 \mathrm{mmHg}$ \\
- WBC count $>12 \mathrm{Gpt} / 1$ or $<4 \mathrm{Gpt} / 1$, or $>10 \%$ immature (band) forms. \\
\hline Sepsis: SIRS caused by infection \\
\hline $\begin{array}{l}\text { Severe Sepsis: Sepsis with at least one organ dysfunction or } \\
\text { hypoperfusion }\end{array}$ \\
\hline $\begin{array}{l}\text { Septic Shock: Severe sepsis associated with hypotension that is resistant } \\
\text { to adequate fluid resuscitation }\end{array}$ \\
Bacteremia: The presence of viable bacteria in the blood stream \\
\hline $\begin{array}{l}\text { Multiple Organ Dysfunction Syndrome (MODS): Impairment of two } \\
\text { or more organ systems in an acutely ill patient where homeostasis cannot } \\
\text { be maintained without therapeutic intervention }\end{array}$
\end{tabular}

Brain involvement is common and plays an increasing role in the management of those critically ill patients in intensive care units (ICU). Therefore, this review gives a 
scientific overview about septic encephalopathy as an important neurological sequela of sepsis.

\section{SEPTIC ENCEPHALOPATHY}

\section{Definition and Clinical Features}

Septic encephalopathy (SE) is defined as brain dysfunction due to sepsis and SIRS [4] and can also be called sepsis-associated delirium. There are no proofed consensus criteria to diagnose SE. It shall only be diagnosed, if any other causes of disturbed brain function are excluded. Extracranial infection and impaired mental state must be evident. Clinically, acute impairment in the level of consciousness and confusion (manifested by alteration in attention, disorientation and concentration up to deep coma in more severe cases) are primarily defining symptoms. Usually it occurs early in the septic process before other organ involvement [5]. It represents the severe end of symptoms like weakness, anorexia, malaise and concentration deficits that are commonly found in febrile patients. Patients may show tremor, myoclonus, asterixis and seldom focal or generalized seizures, whereas those symptoms are seen less often in SE than in metabolic encephalopathies. Neurological findings are symmetrical and rigidity is common [6-8]. Newly developed focal neurological deficits misfit to $\mathrm{SE}$ and require further diagnostics, e.g. brain imaging [9].

\section{Epidemiology and Prognosis}

$\mathrm{SE}$ is still poorly investigated and epidemiological data vary depending on the definition used. However, SE is acknowledged as the most common encephalopathy in intensive care units (ICU). $9-71 \%$ of patients with sepsis develop SE [7, 10-12]. Patients with known CNS pathology are at greater risk for SE. Whereas SE is often described as a reversible syndrome, actually new studies showed prolonged cognitive impairment and depressive symptoms in sepsis survivors [4]. Usually, patient's level of consciousness rapidly improves with treatment of the underlying sepsis. Nevertheless, mortality in SE is high and correlates with severity of septic encephalopathy, e.g. a $63 \%$ mortality rate was reported in SE patients with reduced Glasgow Coma Scale to $3-8$ points [11].

\section{Pathophysiology}

The SE pathogenesis is likely to be multifactorial (Table 2) and is still poorly understood. Most studies investigating SE pathophysiology used animals or cell cultures. Therefore, interpretation for humans must be done with caution.

One important factor leading to cognitive impairment in $\mathrm{SE}$ is oxidative stress. CNS oxidative stress occurs early $(<6 \mathrm{~h})$ after experimental sepsis induction. Interestingly, there was no oxidative damage in brain at later time points (12$96 \mathrm{~h}$ ) in contrast to other organs like lung, liver, heart or kidneys, corresponding well with the usual clinical course [4].

Furthermore, different cytokines, chemokines and inflammatory mediators particularly contribute to the pathogenesis of SE. Pro-inflammatory cytokines (tumor necrosis factor (TNF)- $\alpha$, Interleukin (IL)-1 $\beta$, IL-6), which represent a systemic response to sepsis, are significantly increased in SE patients compared with septic patients without SE and could contribute to the development of longterm cognitive dysfunction and behavioral symptoms. This phenomenon also accounts for increased prevalence of depression in critically ill people $[13,14]$. Nevertheless, some cytokines like IL-6 have additional anti-inflammatory qualities and may be involved in the biphasic course of systemic inflammatory response.

The complement cascade also plays a role in the pathogenesis of SE. Excessive complement activation can also produce reactive oxygen species, can facilitate proinflammatory mediators and causes edema, cell necrosis or apoptosis $[15,16]$.

In addition, diminished cholinergic innervation in hippocampal, parietal and prefrontal cortex is shown after experimental sepsis in rats, probably related to incapacities of memory functions [17]. Dysfunction of the neural circuits between medial temporal lobe, posterior parietal cortex and dorsal prefrontal cortex by disconnection from general activating projections from the ascending reticular activating system (ARAS) in the brainstem may be caused by apoptosis and de-novo atrophy and may cause delirium and long-term impairment in SE patients [18]. Also autonomic nervous and neuroendocrine systems influence immunity, and brain dysfunction can result in a vicious circle with final organ failure $[19,20]$.

Important factors involve cerebral endothelial dysfunction, microvasculature and blood-brain-barrier (BBB) changes leading to impaired nutrition delivery and removal of metabolic waste products as well as increased permeability for substances that are tightly controlled in normal conditions [7]. In terms of increased BBB permeability, elevated protein content in CSF is reported in patients with severe SE [21], which is in accordance with experimental data showing a correlation of cytokine administration and $\mathrm{BBB}$ disruption [22]. Overexpression of inducible nitric oxide synthase (iNOS) in endothelial cells increases BBB permeability [23]. Cerebrovascular endothelial cell pinocytosis is increased in sepsis allowing immunologically relevant molecules to pass through the $\mathrm{BBB}$, nevertheless intercellular tight junctions remain morphologically intact and maintain probably a barrier to inflammatory cells like leukocytes [24]. In addition, cerebrovascular endothelium cells express low levels of leukocyte adhesion molecules, e.g. ICAM-1 and VCAM [25] impeding cerebral leukocyte invasion. However, activated leukocytes generate oxygen free radicals reacting with erythrocytes of septic patients leading to reduced erythrocyte deformability and increased red blood cell aggregation [26, 27]. This phenomenon may contribute to the known cerebral hypoperfusion in SE patients [28]. Perimicrovascular edema diminish diffusion and hence utilization of oxygen and nutrients as well as depletion of waste products in sepsis animal models, too [29]. Also in humans, a reduction of cerebral blood flow has been found during endotoxic shock and could not be improved by elevated mean arterial blood pressure, probably due to this edema [28] and consecutive intracranial hypertension. Additionally, the adrenergic system has been implicated in the inflammatory response to sepsis and may control blood-brain barrier permeability [22]. B2-adrenoceptor stimulation decreases endothelial cell permeability in vitro [30]. The $\beta 2$-adrenoceptor agonist 
dopexamine inhibits perimicrovessel edema formation experimentally, whereas the $\alpha 1$-adrenoceptor agonist methoxamine provokes it [31]. Interestingly, failure of microvasculature with neurovascular uncoupling seems to precede functional changes (e.g. SEPs) and effects on systemic circulation in animal models [15, 32]. Since cerebral autoregulation is disturbed in SE [33], blood pressure drops during sepsis may directly be transferred to the cerebral vascular bed and lead to cerebral hypoperfusion. Therefore, inappropriate blood supply to neurons may play an important role for SE. On the other hand, also hypertensive episodes could cause encephalopathy in a septic brain with autoregulation disturbance (especially in the posterior circulation), which then would lead to the clinical and radiological picture of posterior reversible encephalopathy syndrome or hypertensive encephalopathy [34].

Another factor may be aromatic amino acids (AAA), normally restricted from the brain by the BBB. AAA are increased in SE patients and seem to correlate with severity of encephalopathy. They may act as false neurotransmitter and/or disturb neurotransmitter synthesis. On the other hand, abnormal systemic metabolism in septic patients may lead to increased serum levels of phenylalanine, ammonia, and tryptophan with influence on procalcitonin and IL-6 levels contributing to SE development [35-37].

Toxin release from microbes is unlikely a relevant factor, because even similar encephalopathies also occur in noninfectious syndromes (e.g. acute pancreatitis) [7]. Therefore, systemic inflammation seems to be even more crucial than infection per se.

Table 2. Important Pathophysiological Mechanisms of Septic Encephalopathy

\begin{tabular}{|l|}
\hline Oxidative stress \\
\hline Pro-inflammatory cytokines \\
\hline Antiinflammatory mediators \\
\hline Complement cascade \\
\hline $\begin{array}{l}\text { Disturbed neural circuits (ARAS, autonomic and neuroendocrine systems, } \\
\text { etc.) }\end{array}$ \\
\hline Apoptosis \\
\hline Endothelial dysfunction \\
\hline Blood-brain-barrier disruption \\
\hline Microvascular failure with neurovascular uncoupling \\
\hline Cerebral autoregulation changes \\
\hline Elevated Aromatic amino acids \\
\hline
\end{tabular}

\section{Diagnostical Features}

There are no diagnostic tests with high specificity for SE. Thus, SE is still a diagnosis of exclusion [7]. Clinical scores may help detecting objective changes of brain functions. Mental status examination can be assessed by the Confusion Assessment Method for the Intensive Care Unit (CAM-ICU) in non-sedated patients, whereby acute onset of changes indicate encephalopathy or rather delirium [38]. Sedation status can be verified by the validated Richmond Agitation-
Sedation Scale (RASS) or Glasgow Coma Scale (GCS) [39]. The Assessment to Intensive Care Environment (ATICE) score enables assessment of awakening, comprehension, and calmness, and relies mostly on eye opening. Reduced ATICE scores $(<10$ of maximum 20) indicate delirium [40]. In addition, in more deeply sedated patients, a clinical approach by assessing brainstem reflexes can help [10].

Electroencephalography (EEG) is the most sensitive diagnostic tool and ranked recordings (normal, excessive theta, predominantly delta, triphasic waves, and suppression or burst suppression) correlate well with SE severity [21]. However, these EEG changes are non-specific as well as reported impairment of sensory evoked potentials (SEP) in $\mathrm{SE}$ [41]. Nevertheless, measurements of short-latency und long-latency-SEP provide a valuable estimation of SE severity [41].

CSF total protein may be elevated in severe SE cases, but cell counts and microbiological cultures remain normal [21]. However, lumbar puncture is irreplaceable to exclude direct infection in suspected meningoencephalitis. Other serum markers as S100B and neuron-specific enolase (NSE) are elevated in adults and children with $\mathrm{SE}$ as well as in other brain injuries and may be helpful in sedated patients [42, 43]. However, these laboratory markers do not correlate with severity of SE [44]. In a small case series in SE patients $\mathrm{S} 100 \mathrm{~B}$ was not increased in CSF despite elevated serum levels. Therefore, an increase of S100B may be due to extracerebral sources and does not proof blood-brain-barrier disruption [45].

\section{Brain Imaging}

Abnormalities in brain imaging are described in septic encephalopathy, but these are scarcely investigated in a systematic manner. CT scans are mostly normal $[12,46]$, however, also white matter hypodensities are reported [47]. MR imaging in a case series of patients with septic shock and acute neurological signs revealed various degrees of leukencephalopathy as well as multiple ischemic strokes [48]. MR findings were associated with patient outcome and patients without MR abnormalities survived without sequelae, while those who died showed clear MRI lesions [48]. Brain damage occurred mainly within the white matter and corresponded to vasogenic edema, probably reflecting blood-brain barrier breakdown. Non-specific white matter lesions are also described in other studies [45, 47] (Fig. 1). Finelli et al. showed bilateral basal ganglia, thalamic, cerebellar, brainstem, and cerebral MR abnormalities in a case report [49]. Whereas the latter study confirmed infarction of basal ganglia secondary to fibrinoid necrosis and thrombosis of small vessels neuropathologically, the former study described predominant perivascular edema originated in Virchow-Robin spaces postmortem [48]. Furthermore, MR abnormalities in terms of a posterior reversible encephalopathy syndrome (PRES) have often been found in patients with severe infection, sepsis or septic shock [50]. MR angiography in this study revealed vasospasm and vessel "pruning", perhaps due to diminished cerebral blood flow. However, some of the SE patients did not show any MRI abnormalities [48]. Thus, specific and reliable imaging parameters do not exist for SE patients so far. Nevertheless, MRI can exclude other cerebral pathologies leading to brain 


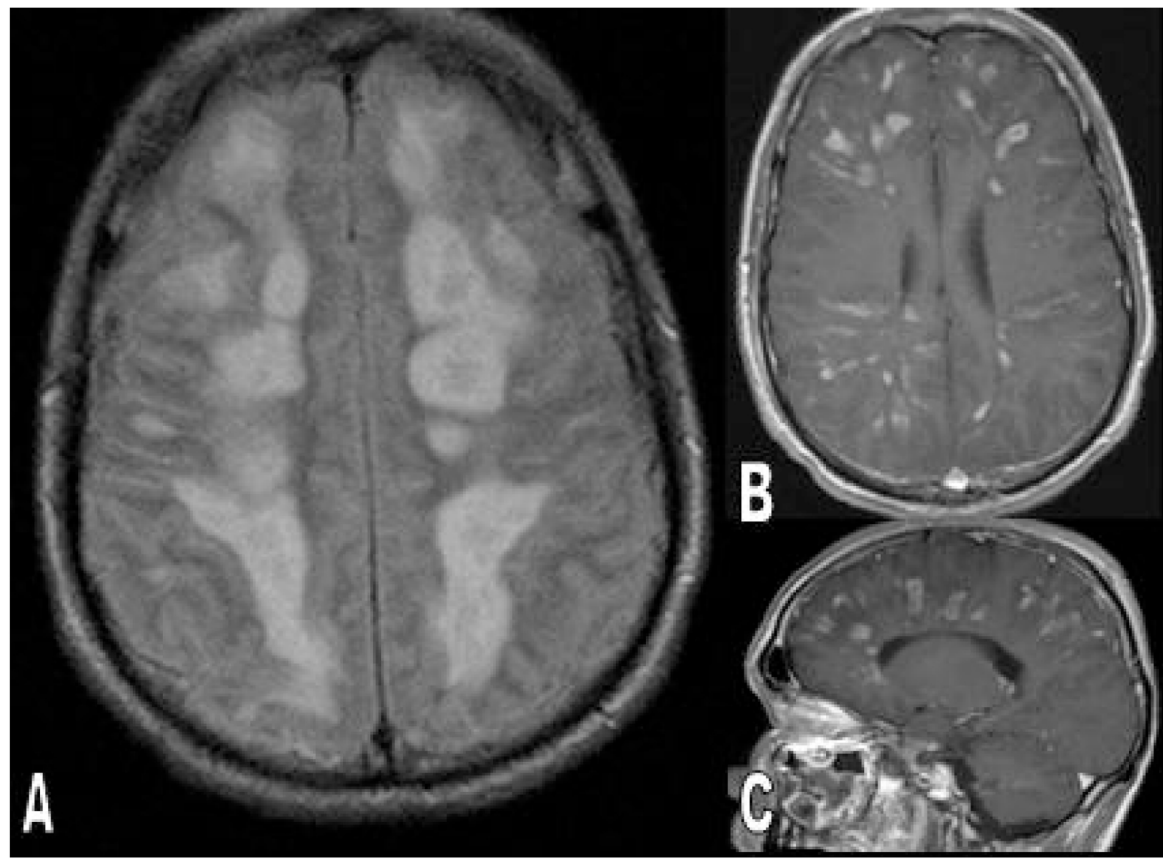

Fig. (1). Axial section of fluid-attenuated inversion recovery weighted (FLAIR) MRI (A), axial (B) and sagittal (C) section post-gadolinium T1-weighted MRI of a patient with severe septic encephalopathy showing confluent white matter lesions with contrast agent enhancement. Courtesy by the Institute of Diagnostic and Interventional Radiology at University Hospital Jena.

dysfunction. We recommend to perform the listed MR sequences (Table 3). Diagnosis of SE may perspectively be facilitated by emerging new MR-sequences, like whole brain spectroscopy or susceptibility weighted imaging (SWI). Therefore, MR imaging is a valuable tool for further studies of SE patients and their underlying pathological processes.

Table 3. Recommendation for MR Imaging Sequences in Suspected SE

Diffusion-weighted (DWI) with calculation of the apparent-diffusioncoefficient (ADC)

Fluid-attenuated inverse recovery (FLAIR)

Suceptibility-weighted echo-planar imaging (EPI-SUS, T2*)

T1-weighted pre- and post-gadolinium exposure (T1post-contrast)

Intracranial time-of-flight MR-angiography (MRA)

Additional perfusion-imaging (PWI) if DWI abnormalities are present

\section{Neuropathological Findings}

There are only a few reports describing neuropathological findings in SE patients (for example, see Fig. 2). Jackson et al. retrospectively showed cerebral hemorrhage, ischemic infarcts and central pontine myelinolysis each in 2 of 12 patients $(\sim 17 \%)$, whereas disseminated micro-abscesses were detectable in 8 of 12 patients $(\sim 67 \%)$ [46]. Similar lesions were described in another study with neuropathological examination of 4 patients, however, their incidence varied greatly and none showed micro-abscesses [51]. In contrast, Sharshar et al. reported ischemic lesions in ischemia-prone regions in all 23 patients which died by septic shock, while microabscesses were seldom found $(9 \%)$. Cerebral hemorrhages were detected comparably in around $26 \%$ of those patients [52] However, this study did not reveal significant differences between septic and non-septic shock in these parameters. In this study only 4 patients, who were not sedated and developed encephalopathy, were included. Small ischemic lesions were seen in all four patients, and a hypercoagulability syndrome or hemorrhages in two of them [52]. Ischemic and apoptotic neurons were found in paraventricular and supraoptic nuclei as well as in locus coeruleus, which are involved in awareness by the noradrenergic brain network. In addition, neuronal lesions were significantly more severe in autonomic system nuclei in septic than in non-septic shock and may contribute to increased SE mortality. Importantly, none of these patients showed septic microabscesses reflecting direct microbial invasion as negligible. Routine and immunohistochemical staining as well as PCR were also negative for bacteria in the prior mentioned study indicating that bacterial infection seems not to be the direct cause of septic appearing infarcts [49]. However, the actual definition of SE was not retained strictly in all of these studies and cases with microabscesses should be classified as focal encephalitis by septic embolization instead of SE. Further studies are required to distinguish between "real" SE and septic embolic encephalitis including brain imaging, prospective clinical and post mortem data.

\section{Therapy}

At present, there are no specific treatment options for SE. Most importantly, adequate and immediate therapy of the underlying sepsis syndrome and supportive intensive care are required. In SE models, there are few promising agents like magnesium, glutamate release inhibitor riluzole or an antioxidant treatment [15]. In future, evolution of selective antagonists of pro-inflammatory cytokine receptors may be a 


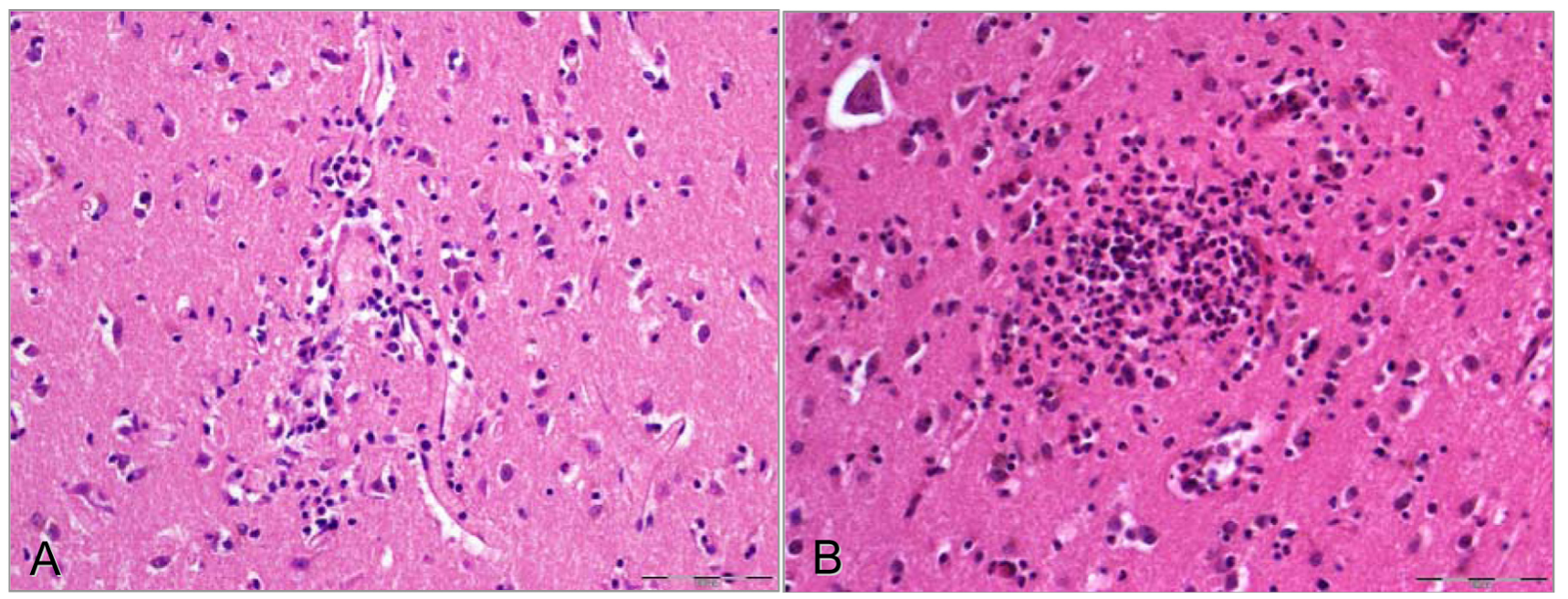

Fig. (2). Example of neuropathological findings (post-mortem sections, H\&E staining): microglial nodule with focal accumulation of activated microglia (A) and micro-abscesses that show much denser accumulation of immune cells including segmented granulocytes (B). However, neuropathological findings are non-specific, and microabscesses should focus suspicion on focal encephalitis.

crucial step to promising therapy options. Alternatively, there are ongoing studies on coupled plasma filtration adsorption, an extracorporal therapy, aimed at the nonspecific removal of cytokines and mediators involved in systemic inflammation - with the goal to reduce early cerebral dysfunction and long-term neurological complications [14].

\section{CONCLUSIVE REMARKS}

Septic encephalopathy or sepsis-associated delirium is characterized by extracranial infection and disturbed mental state without any causes of brain dysfunction except of the underlying SIRS. SE is the most common encephalopathy in ICU patients, nevertheless, it is still often underestimated and frequently not diagnosed. Precise and regular clinical evaluation of brain function in ICU patients is crucial, although reliable clinical diagnostic methods remain to be defined. Electrophysiologically, EEG and SEP add important information and correlate with SE severity. Imaging is necessary in cases with new focal neurological deficits. MRI can thus exclude different etiologies, as focal septic encephalitis, and may unmask underlying leukencephalopathy. However, brain MRI abnormalities and neuropathological findings vary considerably, which may be explained by the multifactorial origin of SE and variable diagnostic criteria used. The pathophysiology is complex and still poorly understood. However, multiple factors including oxidative stress, inflammatory mediators, endothelial dysfunction, blood-brain-barrier disruption, microvascular changes, cerebral autoregulation limitations, excitatory amino-acids, as well as changes of neurotransmitters and dysfunctions of neural circuitry may account for cerebral disturbances. Direct microbial invasion may not play a predominant role, and septic embolization should be differentiated as focal septic encephalitis due to direct intracerebral infection. Thus, systemic inflammation seems to be even more crucial than infection per se in septic encephalopathy. Specific therapy strategies are currently not available. Adequate therapy of the underlying sepsis syndrome and supportive intensive care are necessary.
Recently, Iwashyna et al. [53] studied the cognitive impairment of older patients with severe sepsis (1194 patients, mean age of survivors 76.9 years), and found the prevalence of moderate to severe cognitive impairment to increase 10.6 percentage points among patients who survived severe sepsis. Therefore, the authors concluded that severe sepsis in this older population was independently associated with substantial and persistent new cognitive impairment and functional disability among survivors [53]. Therefore, increasing demand exists for clinical studies in order to clarify the pathophysiology and clinical course of SE and to develop extended therapeutic options in the future.

\section{ACKNOWLEDGEMENTS}

This work was supported by the Center of Sepsis Control\&Care (CSCC), funded by the German Federal Ministry of Education and Research (BMBF) grant no. 01 E0 1002.

\section{REFERENCES}

[1] ACCP/SCCM, American College of Chest Physicians/Society of Critical Care Medicine Consensus Conference. Definitions for sepsis and organ failure and guidelines for the use of innovative therapies in sepsis. Crit Care Med 1992; 20: 864-76.

[2] Levy MM, Fink MP, Marshall JC, et al. 2001 SCCM/ESICM/ACCP/ATS/SIS International Sepsis Definitions Conference. Crit Care Med 2003; 31(4): 1250-6.

[3] Robertson CM, Coopersmith CM. The systemic inflammatory response syndrome. Microbes Infect 2006; 8(5): 1382-9.

[4] Streck EL, Comim CM, Barichello T, Quevedo J. The septic brain Neurochem Res 2008; 33(11): 2171-7.

[5] Bolton CF, Young GB, Zochodne DW. The neurological complications of sepsis. Ann Neurol 1993; 33(1): 94-100.

[6] Weathers AL, Lewis SL. Rare and unusual ... or are they? Less commonly diagnosed encephalopathies associated with systemic disease. Semin Neurol 2009; 29(2): 136-53.

[7] Davies NW, Sharief MK, Howard RS. Infection-associated encephalopathies: their investigation, diagnosis, and treatment. J Neurol 2006; 253(7): 833-45.

[8] Young GB. Neurologic complications of systemic critical illness. Neurol Clin 1995; 13(3): 645-58.

[9] Hund E. Septic encephalopathy. Dtsch Med Wochenschr 2007; 132(7): 322-4.

[10] Ebersoldt M, Sharshar T, Annane D. Sepsis-associated delirium Intens Care Med 2007; 33(6): 941-50. 
[11] Eidelman LA, Putterman D, Putterman C, Sprung CL. The spectrum of septic encephalopathy. Definitions, etiologies, and mortalities. JAMA 1996; 275(6): 470-3.

[12] Young GB, Bolton CF, Austin TW, Archibald YM, Gonder J, Wells GA, The encephalopathy associated with septic illness. Clin Invest Med 1990; 13(6): 297-304.

[13] Dantzer R, O'Connor JC, Freund GG, Johnson RW, Kelley KW. From inflammation to sickness and depression: when the immune system subjugates the brain. Nat Rev Neurosci 2008; 9(1): 46-56.

[14] Wratten ML. Therapeutic approaches to reduce systemic inflammation in septic-associated neurologic complications. Eur $\mathbf{J}$ Anaesthesiol Suppl 2008; 42: 1-7.

[15] Pytel P, Alexander JJ. Pathogenesis of septic encephalopathy. Curr Opin Neurol 2009; 22(3): 283-7.

[16] Jacob A, Hensley LK, Safratowich BD, Quigg RJ, Alexander JJ. The role of the complement cascade in endotoxin-induced septic encephalopathy. Lab Invest 2007; 87(12): 1186-94.

[17] Semmler A, Frisch C, Debeir T,et al. Long-term cognitive impairment, neuronal loss and reduced cortical cholinergic innervation after recovery from sepsis in a rodent model. Exp Neurol 2007; 204(2): 733-40.

[18] Gunther ML, Jackson JC, Ely EW. Loss of IQ in the ICU brain injury without the insult. Med Hypotheses 2007; 69(6): 1179-82.

[19] Chrousos GP. The hypothalamic-pituitary-adrenal axis and immune-mediated inflammation. N Engl J Med 1995; 332(20): 1351-62.

[20] Annane D, Trabold F, Sharshar T, et al. Inappropriate sympathetic activation at onset of septic shock: a spectral analysis approach. Am J Respir Crit Care Med 1999; 160(2): 458-65.

[21] Young GB, Bolton CF, Archibald YM, Austin TW, Wells GA. The electroencephalogram in sepsis-associated encephalopathy. J Clin Neurophysiol 1992; 9(1): 145-52.

[22] Davies DC. Blood-brain barrier breakdown in septic encephalopathy and brain tumours. J Anat 2002; 200(6): 639-46.

[23] Mayhan WG. Effect of lipopolysaccharide on the permeability and reactivity of the cerebral microcirculation: role of inducible nitric oxide synthase. Brain Res 1998; 792(2): 353-7.

[24] Papadopoulos MC, Davies DC, Moss RF, Tighe D, Bennett ED. Pathophysiology of septic encephalopathy: a review. Crit Care Med 2000; 28(8): 3019-24.

[25] dos Santos WL, Rahman J, Klein N, Male DK. Control of lymphocyte adhesion to brain and aortic endothelium: ICAM-1, VCAM-1 and negative charge. J Neuroimmunol 1996; 66(12):125-34

[26] Machiedo GW, Powell RJ, Rush BF, Jr. Swislocki NI, Dikdan G. The incidence of decreased red blood cell deformability in sepsis and the association with oxygen free radical damage and multiplesystem organ failure. Arch Surg 1989; 124(12): 1386-9.

[27] Baskurt OK, Gelmont D, Meiselman HJ. Red blood cell deformability in sepsis. Am J Respir Crit Care Med 1998; 157(2): 421-7.

[28] Maekawa T, Fujii Y, Sadamitsu D, et al. Cerebral circulation and metabolism in patients with septic encephalopathy. Am J Emerg Med 1991; 9(2): 139-43.

[29] Papadopoulos MC, Lamb FJ, Moss RF, Davies DC, Tighe D, Bennett ED. Faecal peritonitis causes oedema and neuronal injury in pig cerebral cortex. Clin Sci (Lond) 1999; 96(5): 461-6.

[30] Zink S, Rosen P, Sackmann B, Lemoine H, Regulation of endothelial permeability by beta-adrenoceptor agonists: contribution of beta 1 - and beta 2-adrenoceptors. Biochim Biophys Acta 1993; 1178(3): 286-98.

[31] Davies DC, Parmar NK, Moss RF, Tighe D, Bennett ED. The role of the adrenergic system in septic encephalopathy. Crit Care 2001; 5(Suppl 1): S83-S84.

[32] Rosengarten B, Hecht M, Auch D, Ghofrani HA, Schermuly RT, Grimminger F, Kaps M. Microcirculatory dysfunction in the brain precedes changes in evoked potentials in endotoxin-induced sepsis syndrome in rats. Cerebrovasc Dis 2007; 23(2-3): 140-7.

[33] Terborg C, Schummer W, Albrecht M, Reinhart K, Weiller C, Rother J. Dysfunction of vasomotor reactivity in severe sepsis and septic shock. Intens Care Med 2001; 27(7): 1231-4.

[34] Schneider JP, Krohmer S, Gunther A, Zimmer C. Cerebral lesions in acute arterial hypertension: the characteristic MRI in hypertensive encephalopathy. Rofo 2006; 178(6): 618-26.

[35] Sprung CL, Cerra FB, Freund HR, et al. Amino acid alterations and encephalopathy in the sepsis syndrome. Crit Care Med 1991; 19(6): 753-7.

[36] Basler T, Meier-Hellmann A, Bredle D, Reinhart K. Amino acid imbalance early in septic encephalopathy. Intens Care Med 2002; 28(3): 293-8

[37] Bleck TP. Neurological disorders in the intensive care unit. Semin Respir Crit Care Med 2006; 27(3): 201-9.

[38] Ely EW, Margolin R, Francis J, et al. Evaluation of delirium in critically ill patients: validation of the Confusion Assessment Method for the Intensive Care Unit (CAM-ICU). Crit Care Med 2001; 29(7): 1370-9.

[39] Ely EW, Truman B, Shintani A, et al. Monitoring sedation status over time in ICU patients: reliability and validity of the Richmond Agitation-Sedation Scale (RASS). JAMA 2003; 289(22): 2983-91.

[40] De Jonghe B, Cook D, Griffith L, et al. Adaptation to the Intensive Care Environment (ATICE): development and validation of a new sedation assessment instrument. Crit Care Med 2003; 31(9): 234454.

[41] Zauner C, Gendo A, Kramer L, et al. Impaired subcortical and cortical sensory evoked potential pathways in septic patients. Crit Care Med 2002; 30(5): 1136-9.

[42] Hsu AA, Fenton K, Weinstein S, Carpenter J, Dalton H, Bell MJ. Neurological injury markers in children with septic shock. Pediatr Crit Care Med 2008; 9(3): 245-51.

[43] Nguyen DN, Spapen H, Su F, et al. Elevated serum levels of S100 beta protein and neuron-specific enolase are associated with brain injury in patients with severe sepsis and septic shock. Crit Care Med 2006; 34(7): 1967-74.

[44] Piazza O, Russo E, Cotena S, Esposito G, Tufano R. Elevated S100B levels do not correlate with the severity of encephalopathy during sepsis. Br J Anaesth 2007; 99(4): 518-21.

[45] Piazza O, Cotena S, De Robertis E, Caranci F, Tufano R. Sepsis associated encephalopathy studied by MRI and cerebral spinal fluid S100B measurement. Neurochem Res 2009; 34(7): 1289-92.

[46] Jackson AC, Gilbert JJ, Young GB, Bolton CF. The encephalopathy of sepsis. Can J Neurol Sci 1985; 12(4): 303-7.

[47] Höllinger P, Zurcher R, Schroth G, Mattle HP. Diffusion magnetic resonance imaging findings in cerebritis and brain abscesses in a patient with septic encephalopathy. J Neurol 2000; 247(3): 232-4.

[48] Sharshar T, Carlier R, Bernard F, et al. Brain lesions in septic shock: a magnetic resonance imaging study. Intens Care Med 2007; 33(5): 798-806.

[49] Finelli PF, Uphoff DF. Magnetic resonance imaging abnormalities with septic encephalopathy. J Neurol Neurosurg Psychiatry 2004; 75(8): 1189-91.

[50] Bartynski WS, Boardman JF, Zeigler ZR, Shadduck RK, Lister J. Posterior reversible encephalopathy syndrome in infection, sepsis, and shock. AJNR Am J Neuroradiol 2006; 27(10): 2179-90.

[51] Bleck TP, Smith MC, Pierre-Louis SJ, Jares JJ, Murray J, Hansen CA. Neurologic complications of critical medical illnesses. Crit Care Med 1993; 21(1): 98-103.

[52] Sharshar T, Annane D, de la Grandmaison GL, Brouland JP, Hopkinson NS, Francoise G. The neuropathology of septic shock. Brain Pathol 2004; 14(1): 21-33.

[53] Iwashyna TJ, Ely EW, Smith DM, Langa KM. Long-term cognitive impairment and functional disability among survivors of severe sepsis. JAMA 2010; 304(16): 1787-94. 